\title{
Theoretical Analysis of the Short Coherence Length Superconductors
}

\author{
K.I. Wysokiński*, A. Ciechan and J. KRZyszczaK \\ Institute of Physics and Nanotechnology Centre, M. Curie-Skłodowska University \\ pl. M. Curie-Skłodowskiej 1, 20-031 Lublin, Poland
}

\begin{abstract}
Properties of the short coherence length $\xi$ superconductors are markedly different from those with long $\xi$. The main difference is connected with their response to impurities. Even the $s$-wave superconductors have been predicted not to obey the so-called Anderson theorem and to show appreciable fluctuations of order parameter. This observation has been recently confirmed in a number of beautiful scanning tunnelling experiments on high temperature cuprate and pnictide superconductors. In this work we study clean two-band systems within BCS-type approach and impure superconductors within mean field Bogolubov-de Gennes approach. In the weak disorder limit both $s$-wave and $d$-wave superconductors only weakly react to impurities. Presence of the Van Hove singularities in the density of states of the two-band clean systems strongly increases their superconducting transition temperature if the intra-band pair scattering is the only pairing interaction. The effect of impurities on such superconductors does depend on the sign of the inter-orbital interaction.
\end{abstract}

PACS numbers: 71.10.Fd, 74.20.-z, 74.81.-g

\section{Introduction}

It is a well recognised fact that impurities present important probes of the superconducting state. The systematic experimental studies of the influence of disorder on superconductivity has been started by Hilsch and Buckel [1] in the early fifties and then continued by many researchers [2]. The theoretical understanding of the relatively weak influence of disorder on classic superconductors was achieved by Anderson 50 years ago [3]. He showed that the BCS prescription for pairing, which requires the occupation of the one-particle eigenstates $|\boldsymbol{k}\rangle$ and $|-\boldsymbol{k}\rangle$ is too restrictive because disorder which breaks translational symmetry should have a deleterious effect on superconductivity in contradiction with many experiments. Anderson showed that, in fact, it is sufficient for members of a Cooper pair to be in states which are time reversed versions of each other and that the effect of disorder is to merely modify the normal metal density of states - the statement which is known as the Anderson theorem for $s$-wave superconductors.

However, it turns out that the Anderson theorem is valid only in the limit of site independent order parameter [4], the assumption which can be shown to be fulfilled in the limit of long coherence length. In this limit the details of disorder are not felt by the members of the Cooper pair. The detailed calculations [4] have predicted the existence of order parameter fluctuations which are

\footnotetext{
* corresponding author; e-mail: karol@tytan.umcs.lublin.pl
}

well characterised by their mean square deviation

$$
\left\langle\left|\delta \Delta_{i}\right|^{2}\right\rangle=\left\langle\left|\Delta_{i}\right|^{2}\right\rangle-\left|\left\langle\Delta_{i}\right\rangle\right|^{2}
$$

where the sign $\langle\ldots\rangle$ denotes the configurational average over impurities configurations. In a system with fluctuating local potentials $\varepsilon_{i}$ assumed to be independent random variables for which $\left\langle\varepsilon_{i} \varepsilon_{j}\right\rangle=\left\langle\varepsilon_{i}^{2}\right\rangle \delta_{i j}$ it has been shown [4] that

$$
\left\langle\left|\delta \Delta_{i}\right|^{2}\right\rangle=\Gamma\left\langle\varepsilon_{i}^{2}\right\rangle .
$$

The coefficient $\Gamma$ depends on the coherence length $\xi$ and vanishes for large $\xi$ indicating that in this limit the Anderson theorem is fulfilled and the fluctuations of the gap vanish. On the contrary, in impure short coherence length superconductors the appreciable gap fluctuations have been predicted. They have been recently discovered in high temperature cuprate (HTS) [5-10] and pnictide superconductors [11-14] and are visible in the scanning tunneling spectroscopy (STS) or microscopy (STM) data.

In HTS the neighbouring regions of the sample show largely different values (by a factor of 2 or 3 ) of the superconducting gap. The gap is defined as the distance between the coherence peaks in the differential conductance. The spread of the gap values in pnictides is smaller and amounts to $\pm 20 \%$.

The description of cuprate superconductors is usually done within $t-J$ or two-component boson-fermion models $[15,16]$. Both of them have successfully been applied to the problem at hand [17-19]. The former model requires assumptions about the changes of pairing interaction energies, but is able to describe the puzzling positive correlation between the position of oxygen dopants and 
the value of the superconducting gap [18]. Similar assumption about the modification of the boson energies which as a result of doping are moved into close vicinity of the Fermi level have to be made in the latter model.

In this paper we discuss three important aspects of disordered short coherence length superconductors using BCS-type theory for clean systems or the Bogolubovde Gennes (BdG) approach for impure ones. The BdG technique correctly treats the inhomogeneities, like grain boundaries, impurities and other defects. The variational wave function used in this method is position dependent. As a result it can appropriately describe the superconduting condensate in a presence of disorder. Contrary to the $\boldsymbol{k}$-space based approaches we do not average over disorder and study the properties of the system with a given configuration of impurities. In the previous studies [20] we have applied similar method to find pairing correlations around two-orbital impurity in a metallic host.

In Sect. 2 we discuss possible increase of the superconducting transition temperature due to the presence of the Van Hove singularities near the Fermi energy in the two-band superconductor with inter-band pairing only. The two-orbital model of impure superconductor is studied in Sect. 3. Section 4 compares the reaction of the $s$-wave and $d$-wave superconductors to impurities. It turns out that in the weak disorder limit this reaction is very much similar for both symmetries, contrary to results of the standard approach which does not allow for a position dependence of the condensate wave function.

\section{Inter-band pairing superconductor $T_{\mathrm{c}}$ enhancement by the Van Hove singularities}

We consider here the model of a clean two-band superconductor, but contrary to the standard approach [21] we assume that the pairing interaction scattering the pairs between different bands is the only active interaction. It reads

$$
H_{\text {pair }}=\sum_{\lambda, \lambda^{\prime}, \boldsymbol{q}, \boldsymbol{k}} U^{\lambda, \lambda^{\prime}} c_{\lambda, \boldsymbol{k}, \uparrow}^{\dagger} c_{\lambda,-\boldsymbol{k}, \downarrow}^{\dagger} c_{\lambda^{\prime},-\boldsymbol{q}, \downarrow} c_{\lambda^{\prime}, \boldsymbol{q}, \uparrow} .
$$

The Cooper pairs are of intra-band character. The interaction $U^{\lambda, \lambda^{\prime}}=U^{12}\left(1-\delta_{\lambda, \lambda^{\prime}}\right)$, however, scatters the pairs between different bands only. Properties of such a model turn out to be vastly different from that with both inter-band and intra-band interactions. The BCS-like equations governing the behaviour of the system read [21]:

$$
\begin{aligned}
& \Delta_{1}=-U_{12} \Delta_{2} \int_{0}^{\hbar \omega_{\mathrm{c}}} \mathrm{d} E N_{2}(E) \frac{\tanh \frac{\sqrt{E^{2}+\Delta_{2}^{2}}}{2 k_{\mathrm{B}} T}}{\sqrt{E^{2}+\Delta_{2}^{2}}}, \\
& \Delta_{2}=-U_{12} \Delta_{1} \int_{0}^{\hbar \omega_{\mathrm{c}}} \mathrm{d} E N_{1}(E) \frac{\tanh \frac{\sqrt{E^{2}+\Delta_{1}^{2}}}{2 k_{\mathrm{B}} T}}{\sqrt{E^{2}+\Delta_{1}^{2}}} .
\end{aligned}
$$

Usually the densities of states in a close vicinity of the Fermi energy are only weakly dependent on energy. In such a case it is a matter of simple calculations to find $[22,23]$ that the ratio $\Delta_{2} / \Delta_{1}=\sqrt{N_{1} / N_{2}}$, where $N_{2}\left(N_{1}\right)$ is the density of states in band 2 (1) at the Fermi level and the superconductiong transition temperature of the system is given by the BCS-like expression [21]:

$$
T_{\mathrm{c}}^{0}=1.136 \frac{\hbar \omega_{\mathrm{c}}}{k_{\mathrm{B}}} \exp \left(\frac{-1}{\lambda_{\text {eff }}}\right)
$$

with $\lambda_{\text {eff }}=\lambda_{0}=\sqrt{U_{12}^{2} N_{1} N_{2}}$.

In a previous paper [24] we have calculated the effect of the Van Hove singularity in the density of states in one of the bands on the $T_{\mathrm{c}}$ and the gap ratio $\Delta_{2} / \Delta_{1}$ and found strong enhancement of them in the limit of small $\lambda_{\text {eff }}$. The first principles calculations of the energy spectrum in the pnictides [25] give two hole-like Fermi pockets centered near the $\Gamma$ point of the Brillouin zone and two, similar in size, electron pockets centered at $M$-points. Due to the nesting properties in the electron and hole Fermi surfaces one expects the singularities in the density of states in both bands.

For the sake of completeness let us assume that they have logarithmic form $[26,27]$ near the Fermi level

$$
N_{\lambda}(E)=N_{\lambda} \ln (2 W /|E|) \Theta(|E|-W)
$$

with $2 W$ being the band width and $\Theta(x)$ - the step function. Solving Eqs. (4) with the density of states given by Eq. (6) near $T_{\mathrm{c}}$ when $\Delta_{1}$ and $\Delta_{2} \rightarrow 0$ one finds (we approximate $\tanh (x)=\min (x, 1)$ and use units in which $\left.\hbar=k_{\mathrm{B}}=1\right)$ :

$$
\begin{aligned}
& \Delta_{1}=-\Delta_{2} U_{12} N_{2}\left[1+\ln \frac{2 W}{\omega_{\mathrm{c}}}+\ln \frac{\omega_{\mathrm{c}}}{2 T_{\mathrm{c}}}\right. \\
& \left.+\ln \frac{2 W}{\omega_{\mathrm{c}}} \ln \frac{\omega_{\mathrm{c}}}{2 T_{\mathrm{c}}}+\frac{1}{2}\left(\ln \frac{\omega_{\mathrm{c}}}{2 T_{\mathrm{c}}}\right)^{2}\right], \\
& \Delta_{2}=-\Delta_{1} U_{12} N_{1}\left[1+\ln \frac{2 W}{\omega_{\mathrm{c}}}+\ln \frac{\omega_{\mathrm{c}}}{2 T_{\mathrm{c}}}\right. \\
& \left.+\ln \frac{2 W}{\omega_{\mathrm{c}}} \ln \frac{\omega_{\mathrm{c}}}{2 T_{\mathrm{c}}}\right] .
\end{aligned}
$$

Here $\omega_{c}$ is the usual BCS energy cut-off. It is easy to notice that in the limit of $\lambda_{\text {eff }} \ll 1$ the above linearised equations lead to

$$
T_{\mathrm{c}}^{2 \mathrm{VH}} \propto \exp \left(-\left(4 / \lambda_{0}\right)^{1 / 4}\right) .
$$

This means a very strong enhancement of the superconducting transition temperature with respect to its value given by Eq. (5). Let us note that the presence of the Van Hove singularity in one of the bands resulted [24] in $T_{\mathrm{c}}^{1 \mathrm{VH}} \propto \exp \left(-\left(2 / \lambda_{0}^{2}\right)^{1 / 3}\right)$. In Fig. 1 we show the dependence of $T_{\mathrm{c}}^{0}, T_{\mathrm{c}}^{2 \mathrm{VH}}$, their ratio $T_{\mathrm{c}}^{2 \mathrm{VH}} / T_{\mathrm{c}}^{0}$ and the ratio $T_{\mathrm{c}}^{1 \mathrm{VH}} / T_{\mathrm{c}}^{0}$ on the coupling $\lambda_{0}$. At low values of $\lambda_{0}$ the $T_{\mathrm{c}}^{0}$ is very low and the Van Hove singularity enhances it by a few orders of magnitude.

\section{Local properties of the two-orbital model}

Our main assumption in the study of the two-orbital model [28] in the context of HTS is that the oxygen impurities are responsible for the appearance of negative $U$ centers inducing superconductivity. The detailed study 


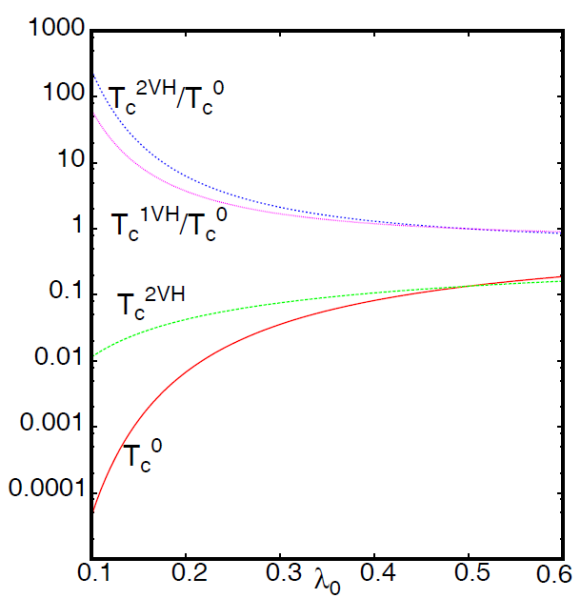

Fig. 1. The coupling constant $\lambda_{0}=\sqrt{U_{12}^{2} N_{1} N_{2}}$ dependence of the superconducting transition temperatures: $T_{\mathrm{c}}^{0}$ of a system with constant density of states, $T_{\mathrm{c}}^{2 \mathrm{VH}}$ of the material with Van Hove singularities in both bands and their ratio $T_{\mathrm{c}}^{2 \mathrm{VH}} / T_{\mathrm{c}}^{0}$. The ratio of a transition temperature of superconductor with Van Hove singularity in one of the bands $T_{\mathrm{c}}^{1 \mathrm{VH}}$ and $T_{\mathrm{c}}^{0}$ is also plotted. Let us note that the Van Hove singularity increases $T_{\mathrm{c}}$ by several orders of magnitude.

shows that the resulting model has a potential to describe some of the correlations observed in the STM experiments [28]. In particular, the short range superconducting interactions allow calculation of the local density of states (LDOS) in sites with $U<0$ and $U=0$. It turns out that LDOS in sites with $U=0$ is characterised by small values of the gap and high well pronounced coherence peaks whereas in sites with $U<0$ the gaps are larger and the coherence peaks wider and lower.

Consider the two-orbital [21] impure superconductor described by the Hamiltonian

$$
\begin{aligned}
H & =\sum_{i j, \lambda \lambda^{\prime}, \sigma}\left[-t_{i j}^{\lambda \lambda^{\prime}}-\mu \delta_{i j} \delta_{\lambda \lambda^{\prime}}+V_{\mathrm{imp}}^{\lambda \lambda^{\prime}}\left(\boldsymbol{r}_{i}\right) \delta_{i j}\right] c_{i \lambda \sigma}^{+} c_{j \lambda^{\prime} \sigma} \\
& +\sum_{i, \lambda \lambda^{\prime}} U_{\lambda \lambda^{\prime}}\left(\boldsymbol{r}_{i}\right) c_{i \lambda \uparrow}^{+} c_{i \lambda \downarrow}^{+} c_{i \lambda^{\prime} \downarrow} c_{i \lambda^{\prime} \uparrow} .
\end{aligned}
$$

In the above equation $c_{i \lambda \sigma}^{+}, c_{i \lambda \sigma}$ denote creation and annihilation operators of electrons with spin $\sigma=\uparrow, \downarrow$ at the lattice site $\boldsymbol{r}_{i}=i$ in the orbital $\lambda . \mu$ is the chemical potential, $t_{i j}^{\lambda \lambda^{\prime}}$ are the hopping integrals between the same (if $\lambda=\lambda^{\prime}$ ) or different orbitals (if $\lambda \neq \lambda^{\prime}$ ) in neighboring sites. $U_{\lambda \lambda^{\prime}}\left(\boldsymbol{r}_{i}\right)$ denotes the on-site interaction, which is attractive for $U_{\lambda \lambda^{\prime}}\left(\boldsymbol{r}_{i}\right)<0$. The dependence of the Hamiltonian parameters on the position $i=\boldsymbol{r}_{i}$ allows a study of inhomogeneous systems.

In the superconducting iron pnictides the role of inter-orbital interactions seem to be of great importance. That is why we concentrate here on the model with $U_{12}$ as the only non-zero interaction. The system contains $15 \%$ impurities randomly distributed over the square lattice of size $19 \times 15$ sites. Impurities are extended as
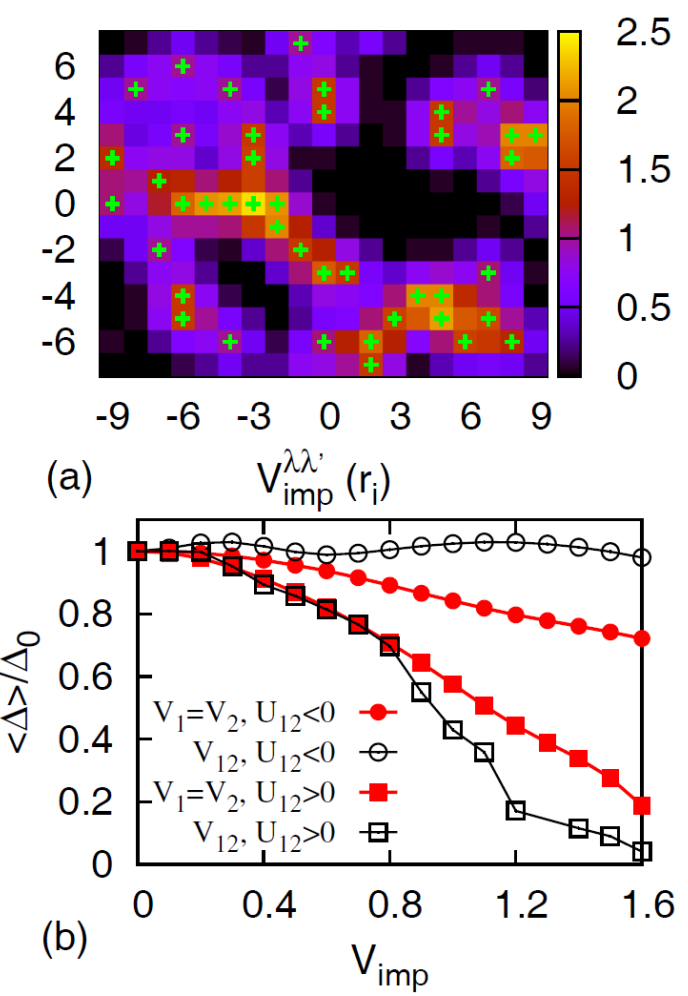

Fig. 2. (a) The distribution of the impurity potential $V_{\mathrm{imp}}^{\lambda \lambda^{\prime}}\left(\boldsymbol{r}_{i}\right)$. (b) The dependence of the normalised mean order parameter on $V_{\mathrm{imp}}=V_{1}=V_{2}$ (bold curves with full symbols) or $V_{\mathrm{imp}}=V_{12}$ (thin curves with empty symbols). The attractive inter-orbital pairing potential $U_{12}=U$ (curves with circles) only weakly affects superconductivity in comparison to repulsive one $U_{12}=-U$ (curves with squares).

$V_{\text {imp }}^{\lambda \lambda \lambda^{\prime}}\left(\boldsymbol{r}_{i}\right)=V_{\lambda \lambda^{\prime}} \sum_{d} f_{i d}$ with $f_{i d}$ being a number from the Gaussian distribution defined in sites at distance $i d=1, \sqrt{2}, 2$ from the impurity. The map presenting the distribution of impurity potentials is shown in Fig. 2a. Figure $2 \mathrm{~b}$ presents the dependence of the mean order parameter $\langle\Delta\rangle / \Delta_{0}$ on the potential strength $V_{\text {imp }}$ for different kinds of impurities and two signs of inter-orbital interaction $U_{12}$. For $U_{12}>0$ impurities suppress the superconducting state much more effectively than for $U_{12}<0$. This is independent of the kind of impurities i.e. if they are of intra-orbital $V_{1}=V_{2}$ or inter-orbital $V_{12}$ character. Weak potential scattering leads to the in-gap bound states for repulsive intra-orbital pairing interaction. For larger scattering strength $V_{12}$, the superconductivity is severely suppressed.

\section{Weakly scattering non-magnetic impurities in the $s$ - and $d$-wave superconductors}

The weak reaction of the superconductor to disorder introduced by means of impurities, alloying, ion beam implantation, amorphisation by quenched condensation, etc. is usually taken as a hint of the $s$-wave symme- 
try of order parameter, and vice versa strong reaction to impurities points towards a non- $s$-wave symmetry. It is the reaction of $\mathrm{Sr}_{2} \mathrm{RuO}_{4}$ to small amount of impurities $[29]$ which was taken as a strong indication of non- $s$-wave symmetry of the order parameter. The influence of non-magnetic impurities on the non- $s$-wave symmetry superconductor $[30,31]$ is similar to the effect of magnetic impurities [32] on superconductor with constant gap. The latter is described by the formula

$$
\ln \left(\frac{T_{\mathrm{c}}}{T_{c_{0}}}\right)=\psi\left(\frac{1}{2}+\frac{\rho}{2}\right)-\psi\left(\frac{1}{2}\right)
$$

with the parameter $\rho=\frac{\hbar}{\pi T_{\mathrm{c}} \tau_{\mathrm{s}}}$ and $\tau_{\mathrm{s}}$ denoting the time for scattering by magnetic impurities. It is proportional to impurity concentration. For anisotropic i.e. $d$ - or $p$-wave superconductors the formula is the same, but the interpretation of the scattering time differs [30, 31]. The relative independence of superconducting transition temperature $T_{\mathrm{c}}$ of $\mathrm{MgB}_{2}$ on $\mathrm{Al}$ or $\mathrm{C}$ impurities, together with its strong degradation by magnetic $\mathrm{Mn}$ impurities is taken as evidence [33] of $s$-wave symmetry of the order parameter in this two-band superconductor.

In high temperature cuprate superconductors believed to be of $d$-wave symmetry the effect of impurities seems to be smaller than expected on the basis of Eq. (10). Here we study the problem by means of the Bogolubovde Gennes theory. We describe the superconductor by the two-component boson-fermion model [34]:

$$
\begin{gathered}
\hat{H}^{\mathrm{BF}}=\sum_{i \sigma}\left(V_{i}^{\mathrm{imp}}-\mu\right) \hat{c}_{i \sigma}^{\dagger} \hat{c}_{i \sigma}+\sum_{i}\left(E_{i}^{\mathrm{B}}-2 \mu\right) \hat{b}_{i}^{\dagger} \hat{b}_{i} \\
\quad+\sum_{i, j, \sigma} t_{i j} \hat{c}_{i \sigma}^{\dagger} \hat{c}_{j \sigma}+\sum_{i, j} \frac{g_{i j}}{2}\left(\hat{b}_{i}^{\dagger} \hat{c}_{i \downarrow} \hat{c}_{j \uparrow}+\hat{b}_{i} \hat{c}_{i \uparrow}^{\dagger} \hat{c}_{j \downarrow}^{\dagger}\right),
\end{gathered}
$$

where $i$ and $j$ denote lattice sites of the two-dimensional system, $\hat{c}_{i, \sigma}$ and $\hat{c}_{i, \sigma}^{\dagger}\left(\hat{b}_{i}\right.$ and $\left.\hat{b}_{i}^{\dagger}\right)$ are fermionic (bosonic) annihilation and creation operators. $\mu$ stands for chemical potential of the whole system (to ensure the charge conservation), $E_{i}^{\mathrm{B}}$ measures the positions of boson energy level and $t_{i j}$ denotes hopping integral. The interaction between fermions and charged bosons is provided by the coupling $g_{i j}$. Its spatial structure defines the symmetry of order parameter. We assume that the potential scattering described by $V_{i}^{\text {imp }}$ is the only source of disorder. The boson energies $E_{i}^{\mathrm{B}}$ are taken to be independent of the site index $i$ and the other parameters in (11) to be periodic functions. The disorder in the two-component model was previously studied for $s$-wave [35] and $d$-wave symmetry order parameter $[19,36]$.

The size of the system is $17 \times 31$ and the other parameters are $t_{1}=1.0 t, E_{i}^{\mathrm{B}}=2 \mu+0.25 t, U_{0}=0.75 t$ and $g_{0}=0.75 t$ in units of $t$. The total carrier concentration $n^{\text {tot }}=n^{\mathrm{f}}+2 n^{\mathrm{b}}=1.3$. The results shown in Fig. 3 indicate that the initial decrease of the superconducting order parameter is nearly the same for both symmetries. Our understanding of this behaviour, which seems to be at odds with standard approach [30], is the following. In the Bogolubov-de Gennes method the $d$-symmetry wave function is defined in real space and is able to distort
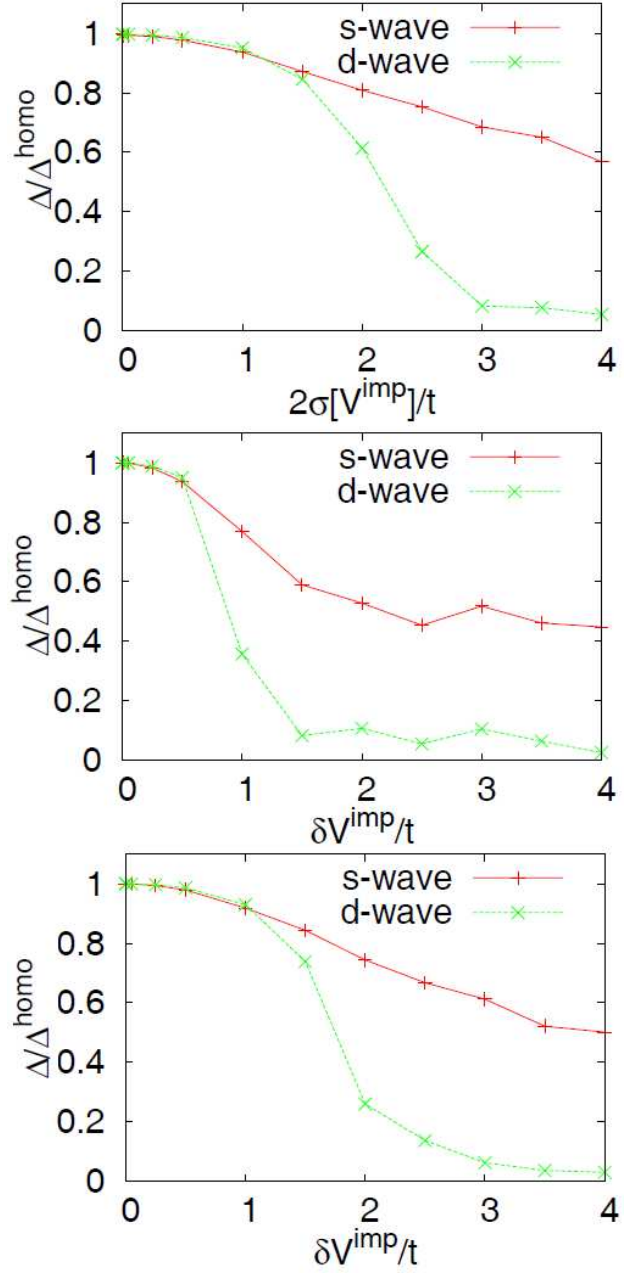

Fig. 3. The effect of the short range potential impurities $V_{i}^{\text {imp }}$ on the averaged value of the superconducting gap $\Delta$ in $s$-wave and $d$-wave symmetry superconductors. Three parts correspond (from the top panel) to Gaussian, bimodal (two-delta distribution), and rectangular disorder distributions, respectively.

around impurities. This means that moderate fluctuations of parameters do not strongly destroy superconductivity globally. In contrast, the conventional approaches average over configurations and assume a uniform order parameter, which is suppressed globally to zero in the critical disorder limit. However, for large disorder the $d$-wave superconducting order vanishes much faster than the $s$-wave one. This interpretation differs from the one presented recently [37].

The dependences shown in Fig. 3 are in agreement with experimental data for some HTS materials [38]. With growing disorder, the distribution of the local order parameters in the system becomes very broad and the average value decreases. At the same time we observe relatively small changes in the total carrier concentration, mostly due to changes in the boson number. The fermion concentration is almost constant in all cases studied. 


\section{Summary and conclusions}

We have studied superconductors with short coherence length described by the two-component or two-orbital models. The fluctuations of the order parameter predicted to exist in such systems [4] are visible in the scanning tunnelling spectroscopy data on both superconducting cuprates and pnictides.

Our main findings can be summarised as follows:

(i) the existence of the Van Hove singularities in the two-band model with inter-band only pairing interaction strongly enhances the superconducting transition temperature,

(ii) the effect of impurities on superconductivity in models with intra-orbital pairing interaction $U^{12}$ depends on its sign. For repulsive $U^{12}$ impurities more strongly degrade $T_{\mathrm{c}}$ than for attractive ones. Similar result has also been obtained [39] within the quasi-classical Eilenberger weak-coupling formalism,

(iii) in the weak disorder limit the changes in the superconducting transition temperature or the superconducting gap (cf. Fig. 3) of $s$-wave and $d$-wave symmetry superconductors are very similar. This is at odds with standard approach based on the perturbative calculations in $\boldsymbol{k}$-space, which predict fast decrease of $T_{\mathrm{c}}$ with disorder for $d$-wave symmetry of the order parameter, but apparently in agreement with some experiments.

\section{Acknowledgments}

The work has been partially supported by the grant no. N N202 187833 . We would like to thank Tadeusz Domański, Roman Micnas, Stanisław Robaszkiewicz and Krzysztof Rogacki for useful discussions.

\section{References}

[1] W. Buckel, R. Hilsch, Z. Phys. 132, 420 (1952); W. Buckel, Physica B 126, 1 (1984).

[2] A.R. Sweedler, D.E. Cox, S. Moehlecke, J. Nucl. Mater. 72, 50 (1978); L.R. Testardi, J.M. Poate, H. Levinstein, Phys. Rev. B 15, 2570 (1977).

[3] P.W. Anderson, J. Phys. Chem. Solids 11, 26 (1959); L.P. Gorkov, Zh. Eksp. Teor. Fiz. 37, 1407 (1959).

[4] B.L. Györffy, G. Litak, K.I. Wysokiński, in: Fluctuation Phenomena in High Critical Temperature Superconducting Ceramics, Eds. M. Ausloos, A.A. Varlamov, Kluwer Academic Publishers NATO ASI Series 1997, p. 385.

[5] T. Cren, D. Roditchev, W. Sacks, J. Klein, J.-B. Moussy, C. Deville-Cavellin, M. Lagues, Phys. Rev. Lett. 84, 147 (2000).

[6] S.-H. Pan, J.P. O'Neal, R.L. Badzey, C. Chamon, H. Ding, J.R. Engelbrecht, Z. Wang, H. Eisaki, S. Uchida, A.K. Gupta, K.-W. Ng, E.W. Hudson, K.M. Lang, J.C. Davis, Nature (London) 413, 282 (2001).

[7] C. Howald, P. Fournier, A. Kapitulnik, Phys. Rev. B 64, 100504(R) (2001).
[8] J.E. Hoffman, E.W. Hudson, K.M. Lang, V. Madhavan, H. Eisaki, S. Uchida, J.C. Davis, Science 295, 466 (2002).

[9] K.M. Lang, V. Madhavan, J.E. Hoffman, E.W. Hudson, H. Eisaki, S. Uchida, J.C. Davis, Nature (London) 415, 412 (2002).

[10] K. McElroy, R.W. Simmonds, J.E. Hoffman, D.-H. Lee, J. Orenstein, H. Eisaki, S. Uchida, J.C. Davis, Nature 422, 592 (2003).

[11] O. Millo, I. Asulin, O. Yuli, I. Felner, Z.-A. Ren, X.-L. Shen, G.-C. Che, Z.-X. Zhao, Phys. Rev. B 78, 092505 (2008).

[12] Y. Yin, M. Zech, T.L. Williams, X.F. Wang, G. Wu, X.H. Chen, J.E. Hoffman, Phys. Rev. Lett. 102, 097002 (2009).

[13] F. Massee, Y. Huang, R. Huisman, S. de Jong, J.B. Goedkoop, M.S. Golden, Phys. Rev. B $\mathbf{7 9}$, 220517(R) (2009).

[14] Yi Yin, M. Zech, T.L. Williams, J.E. Hoffman, Physica C: Superconductivity 469, 535 (2009).

[15] P.W. Anderson, Science 235, 1196 (1987).

[16] R. Micnas, J. Ranninger, S. Robaszkiewicz, Rev. Mod. Phys. 62, 113 (1990).

[17] T.S. Nunner, B.M. Andersen, A. Melikyan, P.J. Hirschfeld, Phys. Rev. Lett. 95, 177003 (2005); B.M. Andersen, A. Melikyan, T.S. Nunner, P.J. Hirschfeld, Phys. Rev. B 74, 060501(R) (2006).

[18] M.M. Maśka, Ż. Śledź, K. Czajka, M. Mierzejewski, Phys. Rev. Lett. 99, 147006 (2007).

[19] J. Krzyszczak, T. Domański, K.I. Wysokiński, R. Micnas, S. Robaszkiewicz, Real Space Inhomogeneities in High Temperature Superconductors: Perspective of the Two-Component Model, preprint, 2009.

[20] A. Ciechan, K.I. Wysokiński, Acta Phys. Pol. A 114 123 (2008).

[21] H. Suhl, B.T. Matthias, L.R. Walker, Phys. Rev. Lett. 3, 552 (1959); M. Suffczynski, Phys. Rev. 128, 1538 (1962).

[22] O.V. Dolgov, I.I. Mazin, D. Parker, A.A. Golubov, Phys. Rev. B 79, 060502(R) (2009).

[23] Y. Bang, H.-Y. Choi, Phys. Rev. B 78, 134523 (2008).

[24] A. Ciechan, K.I. Wysokiński, Local Spectra of Two-Band Model with Intra- and/or Interband Pairs, preprint, 2009.

[25] I.I. Mazin, D.J. Singh, M.D. Johannes, M.H. Du, Phys. Rev. Lett. 101, 057003 (2008).

[26] J. Labbe, J. Bok, Europhys. Lett. 3, 1225 (1987).

[27] R.S. Markiewicz, J. Phys. Chem. Solids 58, 1179 (1997).

[28] A. Ciechan, J. Krzyszczak, K.I. Wysokiński, J. Phys. Conf. Series 150, 052283 (2009).

[29] A.P. Mackenzie, Y. Maeno, Rev. Mod. Phys. 75, 657 (2003).

[30] A.I. Larkin, JETP Lett. 2, 130 (1965).

[31] A.M. Martin, G. Litak, B.L. Gyorffy, J.F. Annett, K.I. Wysokiński, Phys. Rev. B 60, 7523 (1999).

[32] L.P. Gor'kov, J. Supercond. 13, 765 (2000). 
[33] K. Rogacki, B. Batlogg, J. Karpinski, N.D. Zhigadlo, G. Schuck, S.M. Kazakov, P. Wägli, R. Puźniak, A. Wiśniewski, F. Carbone, A. Brinkman, D. van der Marel, Phys. Rev. B 73, 174520 (2006).

[34] J. Ranninger, S. Robaszkiewicz, Physica B 135, 468 (1985); S. Robaszkiewicz, R. Micnas, J. Ranninger, Phys. Rev. B 36, 180 (1987).

[35] G. Pawłowski, S. Robaszkiewicz, Physica A 299, 475 (2001); G. Pawłowski, S. Robaszkiewicz, Mol. Phys. Rep. 34, 76 (2001); G. Pawłowski, S. Robaszkiewicz, Phys. Status Solidi B 236, 400 (2003); S. Robaszkiewicz, G. Pawłowski, J. Supercond. 17, 37 (2004); G. Pawłowski, S. Robaszkiewicz, R. Micnas, J. Supercond. 17, 33 (2004); G. Pawłowski, S. Robaszkiewicz, R. Micnas, Acta Phys. Pol. A 106, 745 (2004).

[36] T. Domański, K.I. Wysokiński, Phys. Rev. B 66, 064517 (2002); T. Domański, J. Ranninger, K.I. Wysokiński, Acta Phys. Pol. B 34, 493 (2003).
[37] A.F. Kemper, D.G.S.P. Doluweera, T.A. Maier, M. Jarrell, P.J. Hirschfeld, H.-P. Cheng, Phys. Rev. B 79, 104502 (2009).

[38] G. Xiao, M.Z. Cieplak, J.Q. Xiao, C.L. Chien, Phys. Rev. B 428742 (1990); S.K. Tolpygo, J.-Y. Lin, M. Gurvitch, S.Y. Hou, J.M. Phillips, Phys. Rev. B 53, 12454 (1996).

[39] V.G. Kogan, C. Martin, R. Prozorov, Phys. Rev. B 80, 014507 (2009). 